\title{
London Dispersion Governs the Interaction Mechanism of Small Polar and Non-Polar Molecules in Metal-Organic Frameworks
}

\author{
Patrick Melix ${ }^{\dagger, \ddagger}$ and Thomas Heine $e^{*,+,}$ \\ $\dagger$ Universität Leipzig, Wilhelm-Ostwald-Institut für Physikalische und Theoretische Chemie, \\ Linnéstraße 2, 04103 Leipzig, Germany. \\ $\ddagger T U$ Dresden, Professur für Theoretische Chemie, Bergstr. 66c, 01062 Dresden, Germany \\ IDepartment of Chemistry, Yonsei University, Seodaemun-gu, Seoul 120-749, Republic of \\ Korea. \\ E-mail: thomas.heine@tu-dresden.de
}

\section{Abstract}

In this work we investigate the adsorption of chlorinated methanes $\left(\mathrm{CH}_{\mathrm{x}} \mathrm{Cl}_{4-\mathrm{x}}, x=\right.$ $0-4)$ in a representative layer-pillar MetalOrganic Framework (MOF), the flexible MOF $\mathrm{Ni}_{2}(\text { ndc })_{2}($ dabco $) \quad($ ndc $=2,6$-naphthalenedicarboxylate, dabco $=1,4$-diazabicyclo-[2.2.2]octane), also known as DUT-8(Ni). The guest molecules show a systematic increase of polarizability with increasing number of chlorine atoms, while the dipole moment exceeds 2 Debye for $x=2$ and 3. Our ligand field molecular mechanics (LFMM) simulations show that, counter-intuitively, the host-guest interactions are mainly characterized by London dispersion, despite the molecular dipole moments reaching magnitudes as large as water. This highlights the importance of London dispersion interactions in the description of host-guest interactions.

\section{Introduction}

A thoroughly investigated representative of breathing flexible Metal-Organic Frameworks (MOFs),${ }^{1-6}$ sometimes also referred to as thirdgeneration $\mathrm{MOFs},{ }^{7-9}$ is the layered pillar $\mathrm{MOF}$ $\mathrm{Ni}_{2}(\text { ndc })_{2}($ dabco $) \quad($ ndc $=2,6$-naphthalene- dicarboxylate, dabco $=1,4$-diazabicyclo- $[2.2 .2]$ octane), also known as DUT-8(Ni). ${ }^{10-27}$ It consists of layers of $\mathrm{Ni}_{2}(\mathrm{COO})_{4}$ paddle wheel nodes interconnected by ndc linkers (Figure 1). The so formed layers are interconnected in the third dimension by dabco pillars (right side of Figure 1).

The flexible form of DUT- $8(\mathrm{Ni})^{23}$ can be switched between two distinct phases: An open pore (op) and a closed pore (cp) phase. The phase transition is triggered by ad- and desorption of guest molecules. We recently presented the possibility to perform molecular dynamics (MD) simulations of DUT8-(Ni) by employing Ligand Field Molecular Mechanics (LFMM) for the description of the metal nodes. ${ }^{25}$ Alzahrani and Deeth lately also confirmed the applicability of LFMM for the description of flexibility in MOFs. ${ }^{29}$

Experimental adsorption energies of chlorinated methanes $\left(\mathrm{CH}_{\mathrm{x}} \mathrm{Cl}_{4-\mathrm{x}}, x=0\right.$ to 3$)$ in DUT-8 $(\mathrm{Ni})(-25$ to $-40,-35$ to -50 , -30 to -40 and -20 to $-40 \mathrm{~kJ} \mathrm{~mol}^{-1}$ for $\mathrm{CCl}_{4}$ to $\mathrm{CH}_{3} \mathrm{Cl}$ respectively, see Figure $\left.2 \mathrm{~b}\right)^{26,30}$ suggest, in agreement with chemical intuition, a significant impact of the guest's dipole moment on the adsorption energy. From the experimental data, a trend relating the adsorption energies with the electrostatic interactions 


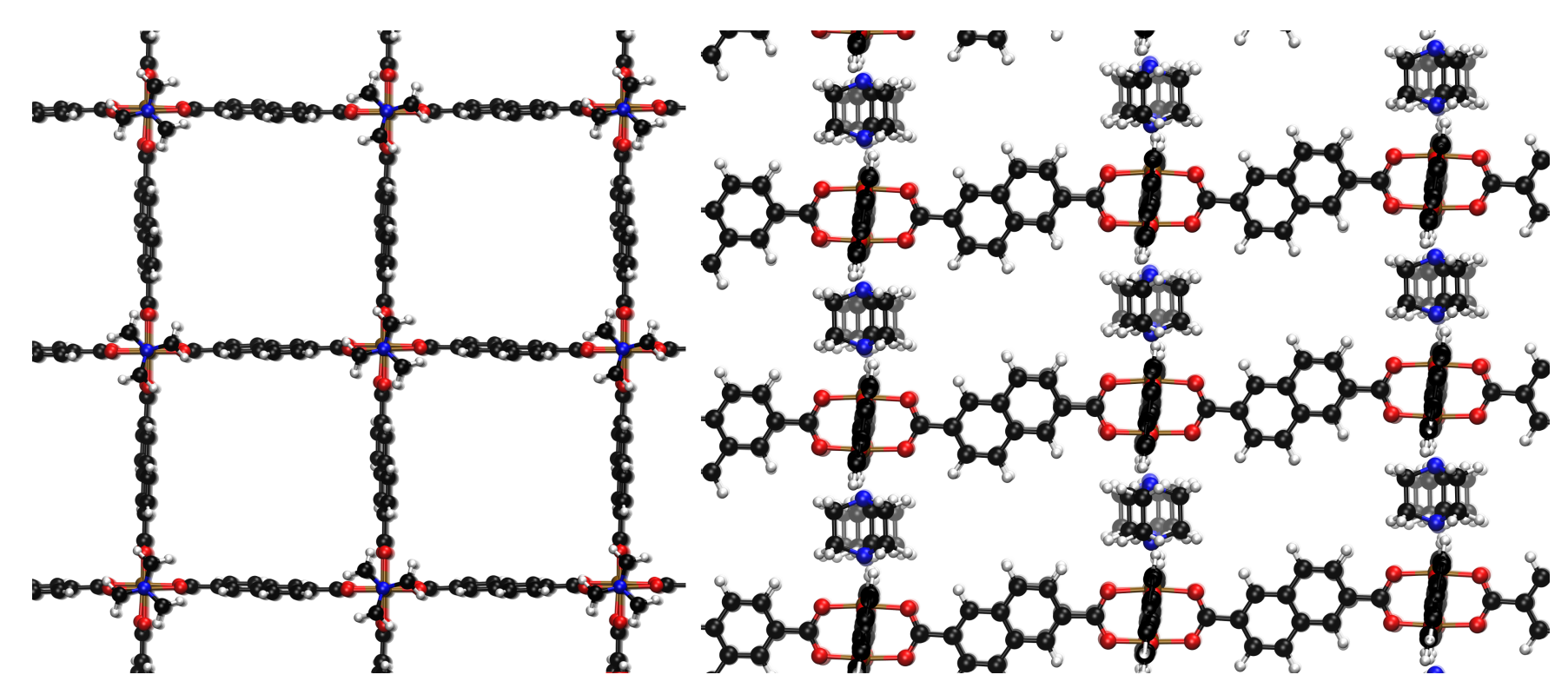

Figure 1: Graphical representation of DUT8-(Ni) conformer $\mathbf{B}^{23}$ in its open phase (op), viewed along the pillar axis (left, $\mathrm{C}$ axis) and along ndc linkers (right, A or B axis). Atoms colored by element: Nickel ochre, oxygen red, nitrogen blue, carbon black and hydrogen white. Reproduced under the terms of the Creative Commons Attribution 4.0 License. ${ }^{28}$

could be suspected (see Figure 2a), even though the reported energy ranges are large. Herein we investigate the contributions of electrostatic and London dispersion interactions upon the adsorption energies of chlorinated methanes $\left(\mathrm{CH}_{\mathrm{x}} \mathrm{Cl}_{4-\mathrm{x}}, x=0-4\right)$ in DUT-8(Ni) by using LFMM simulations. For the case of methane, no experimental adsorption energy is available yet, so we predict its value herein.

The number of theoretical studies of guests in fully flexible DUT-8(Ni) is, to our knowledge, limited to our previous works, ${ }^{23,25}$ probably due to the challenges of correctly modelling the Nickel nodes. However, given the available experimental adsorption data of $\mathrm{CH}_{\mathrm{x}} \mathrm{Cl}_{4-\mathrm{x}}$ molecules, it is a suitable benchmark system to test the adsorption properties without loss of generality. Experimentally different conditions for the adsorption of methane have been reported: Opening of the structure is observed at $111 \mathrm{~K}$ and 1 bar methane pressure, ${ }^{30}$ but at $195 \mathrm{~K}$ and up to 2 bar no transition occurs. ${ }^{22}$ All chlorinated methanes are able to switch the framework from the $\mathbf{c p}$ to the op phase. ${ }^{26,30}$ An important factor for the capability to switch the MOF from cp to op, besides the energy gain, is the kinetic diameter of the guest (see Figure $2 \mathrm{a})$. The kinetic diameter is also connected to the maximum loading, which in turn affects the total adsorption energy gain. Large diameters kinetically hinder the guest in entering the $\mathbf{c p}$ phase. Since we are mainly concerned with energetic arguments in this work, we will refrain from reproducing the kinetic arguments already made. ${ }^{26,30}$

\section{Theoretical Methods}

We used LFMM parameters derived from density functional reference data for the description of the Nickel coordination as reported in ref [25]. Ligands and pillars are described using the general amber force field (GAFF), ${ }^{31}$ which we also employed for all guest molecules. Atomic charges of the MOF framework have been taken from ref [25] (calculated using the CM5 methodology). Atomic charges of the guest molecules were calculated using the ChElPG formalism ${ }^{32}$ implemented in Gaussian09 ${ }^{33}$ using the TPSSh functional and a TZVP basis. Further details are available in Section 1.1 of the SI. An extended DL_POLY Classic software ${ }^{34,35}$ was used for MD simulations. The NoT ensemble was used by employing a Nose-Hoover thermostat and a Hoover 

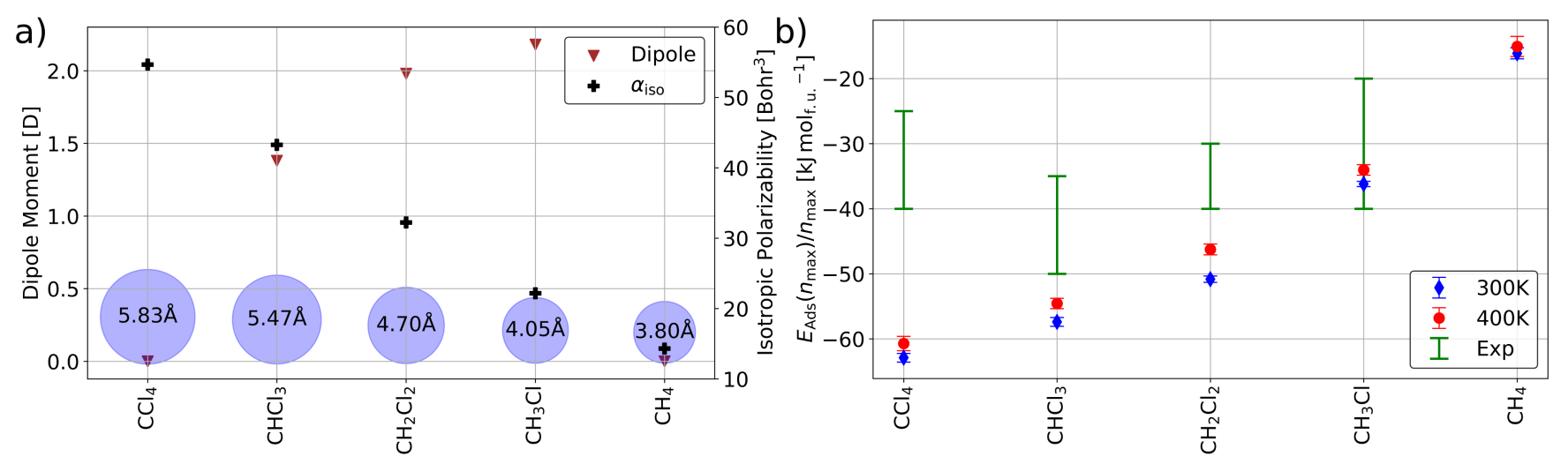

Figure 2: (a) Dipole moments (brown triangles, left scale) and isotropic polarizabilities (blue crosses, right scale) of chlorinated methanes and methane. Kinetic diameters are represented by circles, with their values as inset. (b) Calculated adsorption energies per guest molecule at full loading (symbols) at $300 \mathrm{~K}$ (blue) and $400 \mathrm{~K}$ (red). Experimental references are given as green ranges if available. ${ }^{26,30}$ Reproduced under the terms of the Creative Commons Attribution 4.0 License. ${ }^{28}$

barostat (pressure of $1 \mathrm{~atm})$. The total simulation time was $1 \mathrm{~ns}$ consisting of $2 \times 10^{6}$ simulation steps with a timestep of $0.5 \mathrm{fs}$. A $4 \times 4 \times 4$ supercell of conformer $\mathbf{B}(\mathbf{o p})$ containing a total of 4224 atoms was used in periodic boundary conditions for simulations. Guest molecules were loaded into the op phase inside spheres of radius $4 \AA$ with the help of the Packmol code. ${ }^{36}$ By employing a stepwise removal routine of the guest molecules (see SI for details) we ensured closing of the structures into the $\mathbf{B}(\mathbf{c l})^{\mathbf{o}}$ conformer. The maximum loading used here should in principle exceed the maximum amount that can be loaded in experiments, since the computational approach is not limited by pore accessibility and partially closed structures. Maximum loadings from experiments and the ones used here are given in Table S3 in the SI. Analysis of the trajectories was performed using the Atomistic Simulation Environment (ASE) Python library. ${ }^{37}$

We separated the total system energy into three parts: The MOF energy $\left(E_{\mathrm{MOF}}\right)$, the guest energy $\left(E_{\text {Guest }}\right)$, and the MOF-guest interaction energy $\left(E_{\mathrm{MOF}-\text { Guest }}\right)$. The former two are obtained by calculating single-point energies on the respective parts of the systems, while the MOF-guest interaction is calculated via $E_{\mathrm{Sys}}=E_{\mathrm{MOF}}+E_{\text {Guest }}+E_{\mathrm{MOF}-\text { Guest }}$. The guest-guest interaction is calculated via $E_{\text {Guest }}=U_{\text {Single-Guest }} * n+E_{\text {Guest-Guest }}+$
$E_{\mathrm{MOF}-G u e s t}$, where $U_{\text {Single-Guest }}$ is the internal energy of a single guest molecule and $n$ is the number of guest molecules per formula unit MOF. The adsorption energy can then be approximated as $E_{\mathrm{Ads}}=E_{\mathrm{MOF}-\text { Guest }}+$ $E_{\text {Guest-Guest. Note that all terms discussed here }}$ are functions of the number of guest molecules $n$ and temperature $T$. Yet we suppressed these here, to improve the readability. We used the unit of energy „kJ $\cdot \mathrm{mol}_{\text {f.u. }}{ }^{-1 \text { ". This should }}$ be read as ",kJ per mol of $\mathrm{Ni}_{2}(\mathrm{ndc})_{2}($ dabco $) \cdot n^{\prime \prime}$, where $n$ is the number of guest molecules as in the equations above. The "mol" therefore references the MOF and not the adsorbed guest. By dividing $E_{\text {Ads }}$ by $n$ we obtain the molar adsorption energy of the guest that can be compared to experimentally obtained values. In all plots, error-bars visualize the standard deviation of the corresponding value. Further details on the force field and MD setups employed can be found in the SI. All input and output files as well as scripts for pre- and postprocessing are available in the published raw data. ${ }^{28}$

\section{Results and Discussion}

Our estimation of adsorption energies assumes an ideal (interaction-free) gas phase of the guest. The calculated heat of adsorption will therefore be slightly overestimated. The adsorption energy consists of the guest-guest in- 
teractions inside the MOF and the MOF-guest interactions. As expected, all MOF-guest and guest-guest interactions are attractive (see Figures $\mathrm{S} 7-10)$.

So far, experimental values of adsorption energies of chlorinated methanes in DUT-8(Ni) are available for the case of a rigid op state. ${ }^{26,30}$ The experimental values were measured using small particles of DUT-8(Ni) (submicron sized and therefore rigid ${ }^{11,18,19,38,39}$ ) at around $300 \mathrm{~K}$ and up to adsorbed volumes of $150 \mathrm{~cm}^{3} \mathrm{~g}^{-1}$. This adsorbed volume corresponds to approximately 4.4 molecules per formula unit (f.u.) MOF (using the ideal gas approximation). Employing the Clausius-Clapeyron equation, the adsorption energies per mole guest (or molar adsorption energies) can be derived from the adsorption isotherms at varying temperatures. We can therefore only compare values around our maximum loading, these are in our nomenclature equal to $E_{\text {ads }}\left(n_{\max }\right) / n_{\max }$. It is important to remember that neither experimental nor calculated values include a contribution from the opening of the $\operatorname{MOF}\left(\Delta E_{\mathrm{MOF}}\right)$. Both the experimental and theoretical values are therefore with reference to the open MOF phase. In Figure $2 \mathrm{~b}$, we present the obtained values for all guests including experimental ranges from references [26, 30].

The calculated adsorption energy of $\mathrm{CH}_{3} \mathrm{Cl}$ agrees well with the experimental reference. For $\mathrm{CH}_{2} \mathrm{Cl}_{2}$ and $\mathrm{CHCl}_{3}$ the deviation is more pronounced, but the trend coincides with the experiments as well. The overall larger energy gain in our simulations can be attributed to the low loading in experiment, where guestguest interactions are expected to be less intense. For $\mathrm{CCl}_{4}$ the suggested trend deviates from the experimental reference. Our calculations predict a significantly larger energy gain than the experiment, even though the loading is much closer to the experimental loading (7 versus approx. 5 molecules per f.u. MOF).

To investigate the slope of the adsorption energy of the chlorinated methanes, we analyzed the contributions of London dispersion interactions (in the following referred to as van der Waals (vdW) interactions) and electrostatic interactions at full loading and $300 \mathrm{~K}$. The same decompositions at $400 \mathrm{~K}$ are given in section 3.7 of the SI (Figures S15 and S16). In Figure $3 \mathrm{a}$ we plotted the vdW contribution ( to the guest-guest and MOF-guest interaction energy for all methane derivates and methane itself. On the right y-axis we gave the calculated isotropic polarizabilities of the respective molecules (for computational details see Section 1.1 of the SI).

As to be expected, each added chlorine atom increases the polarizability and therefore the amount of vdW interaction between the guests and between guest and MOF. From $\mathrm{CH}_{4}$ to $\mathrm{CCl}_{4}$ the decrease of $E_{\mathrm{vdW}}$ is almost linear for both interactions. Only the $\mathrm{CCl}_{4}$ contributions deviate slightly from a linear description. To investigate the accuracy of the guest-guest interactions in our simulations, we performed MP2 calculations (Def2QZVPP basis with counterpoise correction) on a $\mathrm{CCl}_{4}$ and a $\mathrm{CHCl}_{3}$ dimer (for details see Section 1.3 of the SI). No significant deviation of the interaction energies was found (less than $3 \mathrm{~kJ} \mathrm{~mol}^{-1}$ difference between MP2 and force field results, see also Section 3.9.1 of the SI). The Radial Distribution Functions (RDF) of carbons and chlorines in $\mathrm{CCl}_{4}$ and $\mathrm{CHCl}_{3}$ explain the slightly lower intermolecular interactions of $\mathrm{CCl}_{4}$ in our simulations: The chlorine atoms in the $\mathrm{CCl}_{4}$ simulations are a little further apart than the ones in the $\mathrm{CHCl}_{3}$ simulations (see Figure S20). We suggest two possible reasons: First, we only applied integer loadings of molecules, it is therefore not surprising that we did not observe an exact match of interatomic chlorine distances since the density relative to the size cannot be exactly matched using integer values of adsorbates. Secondly, $\mathrm{CCl}_{4}$ does not have any dipole. It therefore misses a slightly attractive contribution that $\mathrm{CHCl}_{3}$ does have.

The electrostatic contributions ( $E_{\text {Elstat }}$ to the interaction energies and dipole moments are given in Figure 3b. In the guest-guest interaction we observe the dipole dependence thought to be relevant.

With increasing magnitude of the dipole, the energy gain from electrostatic interactions increases. The MOF-guest interaction does not follow the trend suggested by the magnitude of 

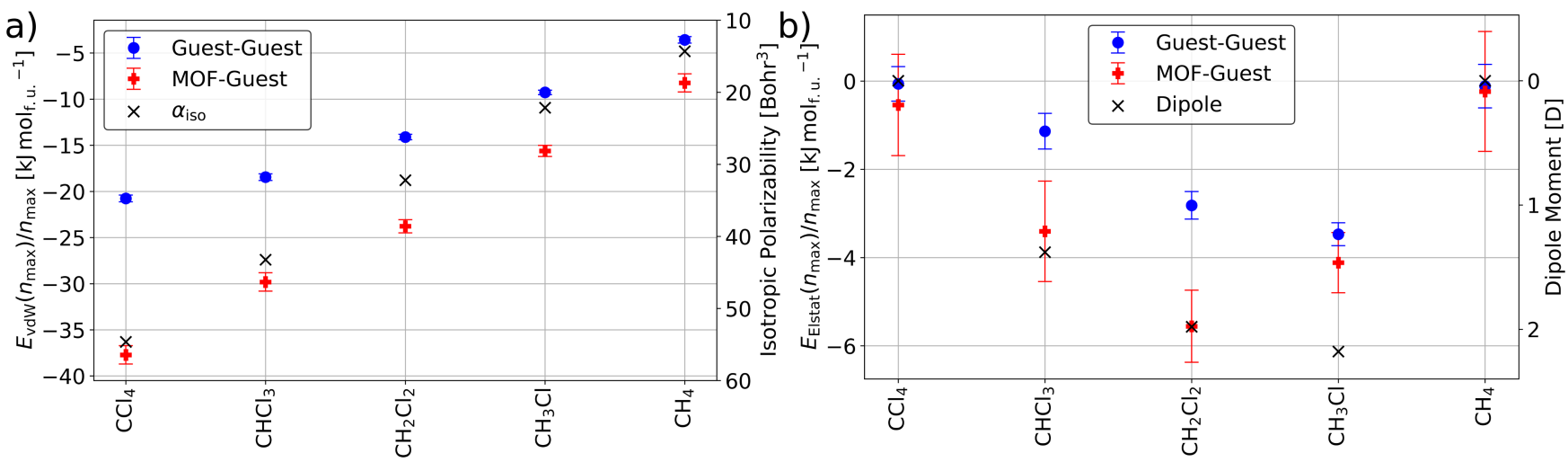

Figure 3: (a) Van der Waals interaction energies of guest-guest and MOF-guest interaction energies per guest molecule at $300 \mathrm{~K}$ and full loading. Calculated isotropic polarizabilities are given on the right-hand y-axis. Note that the polarizability axis is inverted to visualize the correct dependence of the interaction energies. (b) Electrostatic energy contribution to the guest-guest (blue) and MOFguest (red) interaction energies per guest molecule at $300 \mathrm{~K}$ and full loading. Dipole moments of the guest from DFT (grey crosses). Note that the dipole is given on an inverted y axis on the right side of the plot to visualize the dependence of the electrostatic interaction on the dipole magnitude. Reproduced under the terms of the Creative Commons Attribution 4.0 License. ${ }^{28}$

the dipole moment for $\mathrm{CH}_{2} \mathrm{Cl}_{2}$ and $\mathrm{CH}_{3} \mathrm{Cl}$. In order to rule out a systematic error of our force field setup we therefore performed a comparison of DFT and our GAFF setup for all methane derivate dimers (see Section 1.4 of the SI). The results (see Table S6) show perfect agreement for both the electrostatic and London dispersion interactions. We therefore rule out a relevant systematic error of our force field setup.

The Coulomb contribution is overall much weaker than the vdW contribution. This means, that following up on our previous work, ${ }^{23}$ the driving force of flexibility in DUT-8 $(\mathrm{Ni})$ upon adsorption of methane derivates are London dispersion interactions. Coming back to the original question of the molar adsorption energy difference of $\mathrm{CCl}_{4}$ between our simulations and the experimental reference, the insights above only decompose the disagreement into its contributions. A possible explanation lies in the limited range of loading used in the experiment. These differences could be analyzed, for example, in adsorption experiments on different MOFs or by simulations on the rigid $\mathrm{A}(\mathrm{op})$ conformer of $\mathrm{DUT}-8(\mathrm{Ni})$.

The trend of the adsorption energies and the corresponding vdW and electrostatic contributions can be rationalized from the magnitudes of the dipoles and the polarizabilities. The validity of our results is also supported by the experimental melting and boiling points of the chlorinated methanes (both rising from $\mathrm{CH}_{4}$ to $\mathrm{CCl}_{4}$, see Table S5 and Figure S3). If the non-existent permanent dipole moment of $\mathrm{CCl}_{4}$ would be a significant factor in the series of guest-guest interactions from $\mathrm{CCl}_{4}$ to $\mathrm{CH}_{3} \mathrm{Cl}$, one would also expect to see a significant deviation from the trend of melting and boiling points. Such a significant deviation is however only observable for $\mathrm{CH}_{4}$ which has neither a significant dispersion contribution nor a permanent dipole moment, confirming the validity of the simulation results.

It could be argued that the low electrostatic interactions are due to the thermal movement of the adsorbate inside the MOF. To assess the influence of guest dynamics on the electrostatic interactions, we calculated autocorrelation functions (ACFs) ${ }^{40}$ of the dipole orientation for each guest molecule (see Section 1.2.5 of the SI for details). At $300 \mathrm{~K}$ only $\mathrm{CH}_{4}$ and $\mathrm{CH}_{3} \mathrm{Cl}$ exhibit a decay of the autocorrelation to below 0.2. All other guests show autocorrelation values of above 0.5 during the 50 ps analyzed (see Section 3.8 of the SI) and are therefore almost frozen in their respective orienta- 
tion. The thermal movement of the guests can therefore not be responsible for the low electrostatic interactions. At $400 \mathrm{~K}$ all guests exhibit autocorrelation values of below 0.2 after 50 ps. In the temperature range used here, we therefore cover almost frozen guest orientations as well as almost liquid-like guest states. By comparing the electrostatic contributions to the adsorption energy at $300 \mathrm{~K}$ and $400 \mathrm{~K}$, we can conclude that the guest dynamics do slightly lower but do not average out the electrostatic interactions. The vdW interactions however are affected by the increased temperature in the same way. Also, the autocorrelation of the guest orientation does not follow the trend of the dipole magnitude but is rather dependent on the bulkiness (not to say diameter because perfectly spherical molecules would probably not exhibit this dependence) or polarizability. The faster decay of the autocorrelation value of $\mathrm{CH}_{3} \mathrm{Cl}$ in comparison to $\mathrm{CH}_{2} \mathrm{Cl}_{2}$ also explains the slightly smaller electrostatic contribution to the MOFguest interaction visible in Figure 3b. Overall, we conclude that the thermal motion of the guest molecules is not responsible for the unintuitive domination of the $\mathrm{vdW}$ interactions.

\section{Conclusion}

In summary, we analyzed the host-guest and guest-guest interactions upon adsorption of chlorinated methanes in DUT-8(Ni). By applying a fully flexible MD setup we were able to cover a significant part of the realistic conformational space and e.g. also capture entropic effects that cannot be modeled using static (e.g. GC) methods. We showed that our simulation model developed previously ${ }^{25}$ allows insights into available experimental results. As for the stability of the MOF itself, dispersion interactions play an important role in the description of the host-guest and guest-guest interactions. Our simulations match experimental heats of adsorption of chlorinated methanes well and call for additional experiments to reinvestigate the heat of adsorption of $\mathrm{CCl}_{4}$. Acknowledgement This project was funded by Deutsche Forschungsgemeinschaft through
FOR2433. Special thanks to our collaborator S. Ehrling for providing insights into experimental procedures and results. We thank ZIH Dresden for providing computational resources.

\section{Supporting Information Avail- able}

- Extended computational details and results not presented in the main text (PDF)

- Raw Data available on Zenodo (DOI: $10.5281 /$ zenodo.3631182) ${ }^{28}$

\section{References}

(1) Li, H.; Eddaoudi, M.; O'Keeffe, M.; Yaghi, O. M. Design and synthesis of an exceptionally stable and highly porous metal-organic framework. Nature 1999, 402, 276-279, DOI: 10.1038/46248.

(2) Serre, C.; Millange, F.; Thouvenot, C.; Noguès, M.; Marsolier, G.; Louër, D.; Férey, G. Very Large Breathing Effect in the First Nanoporous Chromium(III)Based Solids: MIL-53 or $\mathrm{Cr}^{\mathrm{III}}(\mathrm{OH}) \cdot \mathrm{O}_{2} \mathrm{C}$ $\mathrm{C}_{6} \mathrm{H}_{4}-\mathrm{CO}_{2} \cdot \mathrm{HO}_{2} \mathrm{C}-\mathrm{C}_{6} \mathrm{H}_{4}-\mathrm{CO}_{2} \mathrm{H}_{x} \cdot \mathrm{H}_{2} \mathrm{O}_{y}$. Journal of the American Chemical Society 2002, 124, 13519-13526, DOI: 10.1021/ja0276974, PMID: 12418906.

(3) Eddaoudi, M. Systematic Design of Pore Size and Functionality in Isoreticular MOFs and Their Application in Methane Storage. Science 2002, 295, 469-472, DOI: $10.1126 /$ science. 1067208.

(4) Kitagawa, S.; Kitaura, R.; Noro, S.i. Functional Porous Coordination Polymers. Angewandte Chemie International Edition 2004, 43, 2334-2375, DOI: 10. 1002/anie. 200300610.

(5) Férey, G.; Serre, C. Large breathing effects in three-dimensional porous hybrid 
matter: facts, analyses, rules and consequences. Chemical Society Reviews 2009, 38, 1380, DOI: 10.1039/b804302g.

(6) Schneemann, A.; Bon, V.; Schwedler, I.; Senkovska, I.; Kaskel, S.; Fischer, R. A. Flexible metal-organic frameworks. Chemical Society Reviews 2014, 43, 6062-6096, DOI: 10.1039/c4cs00101j.

(7) Kitagawa, S.; Kondo, M. Functional Micropore Chemistry of Crystalline Metal Complex-Assembled Compounds. Bulletin of the Chemical Society of Japan 1998, 71, 1739-1753, DOI: 10.1246/bcsj.71. 1739

(8) Horike, S.; Shimomura, S.; Kitagawa, S. Soft porous crystals. Nature Chemistry 2009, 1, 695-704, DOI: 10.1038/nchem. 444.

(9) Liu, J.; Chen, L.; Cui, H.; Zhang, J.; Zhang, L.; Su, C.-Y. Applications of metal-organic frameworks in heterogeneous supramolecular catalysis. Chemical Society Reviews 2014, 43, 6011-6061, DOI: $10.1039 / c 4 c s 00094 c$.

(10) Klein, N.; Herzog, C.; Sabo, M.; Senkovska, I.; Getzschmann, J.; Paasch, S.; Lohe, M. R.; Brunner, E.; Kaskel, S. Monitoring adsorption-induced switching by ${ }^{129} \mathrm{Xe}$ NMR spectroscopy in a new metal-organic framework $\mathrm{Ni}_{2}(2,6 \text {-ndc) })_{2}$ (dabco). Physical Chemistry Chemical Physics 2010, 12, 11778, DOI: $10.1039 / \mathrm{c} 003835 \mathrm{k}$.

(11) Lee, J. Y.; Pan, L.; Huang, X.; Emge, T. J.; Li, J. A Systematic Approach to Building Highly Porous, Noninterpenetrating Metal-Organic Frameworks with a Large Capacity for Adsorbing $\mathrm{H}_{2}$ and $\mathrm{CH}_{4}$. Advanced Functional Materials 2011, 21, 993-998, DOI: 10.1002/adfm. 201001790.

(12) Hoffmann, H. C.; Assfour, B.; Epperlein, F.; Klein, N.; Paasch, S.; Senkovska, I.; Kaskel, S.; Seifert, G.;
Brunner, E. High-Pressure in Situ ${ }^{129} \mathrm{Xe}$ NMR Spectroscopy and Computer Simulations of Breathing Transitions in the Metal-Organic Framework $\mathrm{Ni}_{2}(2,6-$ ndc) ${ }_{2}$ (dabco) (DUT-8(Ni)). Journal of the American Chemical Society 2011, 133, 8681-8690, DOI: 10.1021/ja201951t, PMID: 21539397.

(13) Klein, N.; Hoffmann, H. C.; Cadiau, A.; Getzschmann, J.; Lohe, M. R.; Paasch, S.; Heydenreich, T.; Adil, K.; Senkovska, I.; Brunner, E.; Kaskel, S. Structural flexibility and intrinsic dynamics in the $\mathrm{M}_{2}(2,6-$ ndc $)_{2}$ (dabco) $(\mathrm{M}=\mathrm{Ni}, \mathrm{Cu}, \mathrm{Co}, \mathrm{Zn})$ metalorganic frameworks. Journal of Materials Chemistry 2012, 22, 10303-10312, DOI: 10.1039/C2JM15601F.

(14) Bon, V.; Klein, N.; Senkovska, I.; Heerwig, A.; Getzschmann, J.; Wallacher, D.; Zizak, I.; Brzhezinskaya, M.; Mueller, U.; Kaskel, S. Exceptional adsorption-induced cluster and network deformation in the flexible metal-organic framework DUT$8(\mathrm{Ni})$ observed by in situ X-ray diffraction and EXAFS. Physical Chemistry Chemical Physics 2015, 17, 17471-17479, DOI: 10.1039/c5cp02180d.

(15) Bon, V.; Kavoosi, N.; Senkovska, I.; Kaskel, S. Tolerance of Flexible MOFs toward Repeated Adsorption Stress. ACS Applied Materials \& Interfaces 2015, 7, 22292-22300, DOI: 10.1021/acsami. 5b05456, PMID: 26397165.

(16) Trepte, K.; Schwalbe, S.; Seifert, G. Electronic and magnetic properties of DUT-8(Ni). Physical Chemistry Chemical Physics 2015, 17, 17122-17129, DOI: 10.1039/c5cp01881a.

(17) Mendt, M.; Gutt, F.; Kavoosi, N.; Bon, V.; Senkovska, I.; Kaskel, S.; Pöppl, A. EPR Insights into Switchable and Rigid Derivatives of the MetalOrganic Framework DUT-8(Ni) by NO Adsorption. The Journal of Physical Chemistry C 2016, 120, 14246-14259, DOI: $10.1021 /$ acs.jpcc.6b04984. 
(18) Kavoosi, N.; Bon, V.; Senkovska, I.; Krause, S.; Atzori, C.; Bonino, F.; Pallmann, J.; Paasch, S.; Brunner, E.; Kaskel, S. Tailoring adsorption induced phase transitions in the pillared-layer type metal-organic framework DUT8(Ni). Dalton Transactions 2017, 46, 4685-4695, DOI: 10.1039/c7dt00015d.

(19) Miura, H.; Bon, V.; Senkovska, I.; Ehrling, S.; Watanabe, S.; Ohba, M.; Kaskel, S. Tuning the gate-opening pressure and particle size distribution of the switchable metal-organic framework DUT-8 $(\mathrm{Ni})$ by controlled nucleation in a micromixer. Dalton Transactions 2017, 46, 14002-14011, DOI: 10.1039/ c7dt02809a.

(20) Krylov, A.; Vtyurin, A.; Petkov, P.; Senkovska, I.; Maliuta, M.; Bon, V.; Heine, T.; Kaskel, S.; Slyusareva, E. Raman spectroscopy studies of the terahertz vibrational modes of a DUT-8 $(\mathrm{Ni})$ metal-organic framework. Physical Chemistry Chemical Physics 2017, 19, 3209932104, DOI: $10.1039 /$ c7cp06225g.

(21) Kavoosi, N.; Savchenko, T.; Senkovska, I.; Maliuta, M.; Bon, V.; Eychmüller, A.; Kaskel, S. Selective pore opening and gating of the pillared layer metal-organic framework DUT-8 $(\mathrm{Ni})$ upon liquid phase multi-component adsorption. Microporous and Mesoporous Materials 2018, 271, 169-174, DOI: 10.1016/j.micromeso. 2018.05 .024 .

(22) Sin, M.; Kavoosi, N.; Rauche, M.; Pallmann, J.; Paasch, S.; Senkovska, I.; Kaskel, S.; Brunner, E. In Situ ${ }^{13} \mathrm{C}$ NMR Spectroscopy Study of $\mathrm{CO}_{2} / \mathrm{CH}_{4}$ Mixture Adsorption by Metal-Organic Frameworks: Does Flexibility Influence Selectivity? Langmuir 2019, 35, 3162-3170, DOI: 10.1021/acs. langmuir.8b03554.

(23) Petkov, P.; Bon, V.; Hobday, C. L.; Kuc, A. B.; Melix, P.; Kaskel, S.; Düren, T.; Heine, T. Conformational isomerism controls collective flexibility in metal-organic framework DUT-8(Ni). Physical Chemistry Chemical Physics 2019, 21, 674-680, DOI: 10.1039/ c8cp06600k.

(24) Mendt, M.; Ehrling, S.; Senkovska, I.; Kaskel, S.; Pöppl, A. Synthesis and Characterization of $\mathrm{Cu}-\mathrm{Ni}$ Mixed Metal Paddlewheels Occurring in the Metal-Organic Framework DUT$8\left(\mathrm{Ni}_{0.98} \mathrm{Cu}_{0.02}\right)$ for Monitoring OpenClosed-Pore Phase Transitions by X-Band Continuous Wave Electron Paramagnetic Resonance Spectroscopy. Inorganic Chemistry 2019, 58, 4561-4573, DOI: 10.1021/acs. inorgchem.9b00123.

(25) Melix, P.; Paesani, F.; Heine, T. A Ligand Field Molecular Mechanics Study of $\mathrm{CO}_{2}{ }^{-}$ Induced Breathing in the Metal-Organic Framework DUT-8(Ni). Advanced Theory and Simulations 2019, 2, 1900098, DOI: 10.1002/adts. 201900098.

(26) Ehrling, S.; Senkovska, I.; Bon, V.; Evans, J. D.; Petkov, P.; Krupskaya, Y.; Kataev, V.; Wulf, T.; Krylov, A.; Vtyurin, A.; Krylova, S.; Adichtchev, S.; Slyusareva, E.; Weiss, M. S.; Büchner, B.; Heine, T.; Kaskel, S. Crystal size versus paddle wheel deformability: selective gated adsorption transitions of the switchable metal-organic frameworks DUT-8(Co) and DUT-8(Ni). Journal of Materials Chemistry A 2019, 7, 2145921475, DOI: 10.1039/c9ta06781g.

(27) Rauche, M.; Ehrling, S.; Krause, S.; Senkovska, I.; Kaskel, S.; Brunner, E. New insights into solvent-induced structural changes of ${ }^{13} \mathrm{C}$ labelled metal-organic frameworks by solid state NMR. Chemical Communications 2019, 55, 9140-9143, DOI: $10.1039 /$ c9cc04298a.

(28) Melix, P. London Dispersion Governs the Interaction Mechanism of Small Polar and Non-Polar Molecules in MetalOrganic Frameworks. Zenodo 2020, DOI: 10.5281/zenodo. 3631182 . 
(29) Alzahrani, K. A. H.; Deeth, R. J. A Computational Analysis of the Intrinsic Plasticity of Five-Coordinate $\mathrm{Cu}$ (II) Complexes and the Factors Leading to the Breakdown of the Orbital Directing Effect in Paddlewheel Secondary Building Units. Journal of Computational Chemistry 2019, 41, 340-348, DOI: 10.1002/ jcc. 26107.

(30) Ehrling, S. Untersuchungen des strukturellen Schaltverhaltens der metallorganischen Gerüstverbindung DUT-8. Ph.D. thesis, Technische Universität Dresden, 2019 .

(31) Wang, J.; Wolf, R. M.; Caldwell, J. W.; Kollman, P. A.; Case, D. A. Development and testing of a general amber force field. Journal of Computational Chemistry 2004, 25, 1157-1174, DOI: 10.1002/jcc. 20035.

(32) Breneman, C. M.; Wiberg, K. B. Determining atom-centered monopoles from molecular electrostatic potentials. The need for high sampling density in formamide conformational analysis. Journal of Computational Chemistry 1990, 11, 361-373, DOI: 10.1002/jcc.540110311.

(33) Inc., G. Gaussian. http://www. gaussian.com.

(34) Todorov, I. T.; Smith, W.; Trachenko, K.; Dove, M. T. DLPOLY3: new dimensions in molecular dynamics simulations via massive parallelism. Journal of $\mathrm{Ma}$ terials Chemistry 2006, 16, 1911, DOI: 10.1039/b517931a.

(35) Cirera, J.; Babin, V.; Paesani, F. Theoretical Modeling of Spin Crossover in MetalOrganic Frameworks: $\left[\mathrm{Fe}(\mathrm{pz})_{2} \mathrm{Pt}(\mathrm{CN})_{4}\right]$ as a Case Study. Inorganic Chemistry 2014, 53, 11020-11028, DOI: 10.1021/ ic501519a.

(36) Martínez, L.; Andrade, R.; Birgin, E. G.; Martínez, J. M. PACKMOL: A package for building initial configurations for molecular dynamics simulations. Journal of Computational Chemistry 2009, 30, 2157-2164, DOI: 10.1002/jcc. 21224.

(37) Larsen, A. H.; Mortensen, J. J.; Blomqvist, J.; Castelli, I. E.; Christensen, R.; Dułak, M.; Friis, J.; Groves, M. N.; Hammer, B.; Hargus, C.; Hermes, E. D.; Jennings, P. C.; Jensen, P. B.; Kermode, J.; Kitchin, J. R.; Kolsbjerg, E. L.; Kubal, J.; Kaasbjerg, K.; Lysgaard, S.; Maronsson, J. B.; Maxson, T.; Olsen, T.; Pastewka, L.; Peterson, A.; Rostgaard, C.; Schiøtz, J.; Schütt, O.; Strange, M.; Thygesen, K. S.; Vegge, T.; Vilhelmsen, L.; Walter, M.; Zeng, Z.; Jacobsen, K. W. The atomic simulation environment - a Python library for working with atoms. Journal of Physics: Condensed Matter 2017, 29, 273002, DOI: $10.1088 / 1361-648 x / a a 680$ e.

(38) Keupp, J.; Schmid, R. Molecular Dynamics Simulations of the Breathing Phase Transformation of MOF Nanocrystallites. Advanced Theory and Simulations 2019, 2, 1900117, DOI: 10.1002/adts . 201900117.

(39) Rogge, S. M. J.; Waroquier, M.; Van Speybroeck, V. Unraveling the thermodynamic criteria for size-dependent spontaneous phase separation in soft porous crystals. Nature Communications 2019, 10, DOI: 10.1038/s41467-019-12754-w.

(40) de Buyl, P. tidynamics: A tiny package to compute the dynamics of stochastic and molecular simulations. Journal of Open Source Software 2018, 3, 877, DOI: 10.21105/joss. 00877 . 